\title{
Universal spatial correlations in random spinor fields
}

\author{
Juan Diego Urbina, ${ }^{1}$ Michael Wimmer, ${ }^{2}$ Dominik Bauernfeind,${ }^{1}$ Diego Espitia, ${ }^{3}$ İnanç Adagideli,${ }^{4}$ and Klaus Richter ${ }^{1}$ \\ ${ }^{1}$ Institut für Theoretische Physik, Universität Regensburg, D-93040 Regensburg, Germany \\ ${ }^{2}$ Instituut-Lorentz, Universiteit Leiden, P.O. Box 9506, 2300 RA Leiden, The Netherlands \\ ${ }^{3}$ Universidad Pedagogica y Tecnologica de Colombia, UPTC, Tunja, Colombia \\ ${ }^{4}$ Faculty of Engineering and Natural Sciences, Sabanci University, Orhanli-Tuzla, 34956 Istanbul, Turkey
}

(Received 12 August 2011; revised manuscript received 12 December 2012; published 17 April 2013)

\begin{abstract}
We identify universal spatial fluctuations in systems with nontrivial spin dynamics. To this end we calculate by exact numerical diagonalization a variety of experimentally relevant correlations between spinor amplitudes, spin polarizations, and spin currents, both in the bulk and near the boundary of a confined two-dimensional clean electron gas in the presence of spin-orbit interaction. We support our claim of universality with the excellent agreement between the numerical results and system-independent spatial correlations of a random field defined on both the spatial and spin degrees of freedom. A rigorous identity relating our universal predictions with response functions provides a direct physical interpretation of our results in the framework of linear response theory.
\end{abstract}

DOI: 10.1103/PhysRevE.87.042115

PACS number(s): 05.60.Gg, 74.20.Fg, 75.10.Jm, 71.10.Li

\section{INTRODUCTION}

The field of spintronics, which deals with the use of the electron spin degree of freedom as means to transmit, store, and process energy and information, has experienced impressive advances during the past decade [1]. The possibility of manipulating spin densities and currents by means of their coupling to the easier-to-control charge (and electrical current) degrees of freedom has been in the focus of semiconductor-based spintronics. Here the spin Hall effect [2], the creation of a spin imbalance across a sample generated by a charge current in the presence of spin-orbit interaction (SOI), is a primary example of the close connection between the spin and charge degrees of freedom and related spatial correlations

In this context, universality of spatial correlations can arise from two basic mechanisms. In systems where the mean free path $l_{f}$ is much smaller than the system size $L$, average over the random distribution of obstacles produces results that are largely independent of the geometry of the confinement. This diffusive limit has been extensively studied using diagramatic techniques based on disorder average [4], which, however, cannot deal with ballistic systems where $l_{f} / L \gg 1$, a regime which is now easily achieved in high-mobility semiconductor 2D electron gases [3]. In this ballistic case, universality arises due to electron scattering with the irregular boundaries, namely, from the presence of classical chaos. It is in this regime where semiclassical approaches to universality [5] in charge transport in the presence of SOI and its breakdown [6] in spin transport have been very successful.

Spatial fluctuations in ballistic spinor systems have been addressed in Ref. [7] in the limit of vanishing SOI, and recently in Ref. [8] the spatial correlations of charge densities for the bulk have been studied by means of random matrix theory. The extension to systems with nonzero local spin polarization (which is realized, for example, by a spin-polarized STM tip effectively performing a projective measurement of the local spin polarization) and in the presence of boundaries requires substantial technical and conceptual steps beyond Refs. [7,8]. Our goal is to fill this gap.
Our starting point is the Schrödinger equation ( $\hat{I}$ is the unit operator in spin space)

$$
\left[\frac{\hat{\mathbf{p}}^{2}}{2 m} \otimes \hat{I}+\frac{\hbar k_{\mathrm{so}}}{m}\left(\hat{p}_{y} \otimes \hat{\sigma}_{x}-\hat{p}_{x} \otimes \hat{\sigma}_{y}\right)\right]\left|\psi_{n}\right\rangle=E_{n}\left|\psi_{n}\right\rangle
$$

with Dirichlet boundary conditions

$$
\psi_{n}(\mathbf{r} \in \partial \Omega, s)=0,
$$

where

$$
\psi_{n}(\mathbf{r}, s)=\left(\left\langle\mathbf{r}|\otimes\langle s|) \mid \psi_{n}\right\rangle .\right.
$$

Equation (1) describes the stationary states of an electron with effective mass $m$ inside a quantum dot with Dirichlet boundary conditions along $\partial \Omega$ in the presence of SOI with inverse precession length $k_{\mathrm{so}}$. The momentum operator $\hat{\mathbf{p}}=$ $-i \hbar(\partial / \partial x, \partial / \partial y)$ in Eq. (1) acts on the orbital degrees of freedom $\mathbf{r}=(x, y)$, and $\left(\hat{\sigma}_{x}, \hat{\sigma}_{y}, \hat{\sigma}_{z}\right)$ are the Pauli matrices acting on the space spanned by the eigenstates of $\hat{\sigma}_{z}$ (denoted by $|s\rangle$ with $s=\uparrow, \downarrow)$.

\section{THE ROLE OF TIME-REVERSAL SYMMETRY}

An important feature of the system described by the Hamiltonian Eq. (1) is the interplay between SOI and time reversal invariance (TRI). TRI is expressed by the condition [9]

$$
\hat{H} \hat{T}=\hat{T} \hat{H},
$$

where

$$
\hat{T}=-i \hat{\sigma}_{y} \hat{K}
$$

is the time reversal operator and $\hat{K}$ indicates complex conjugation in the basis $|\uparrow\rangle,|\downarrow\rangle$.

For arbitrary $k_{\mathrm{so}}$, eigenstates of $\hat{H}$ come in degenerate (Kramers) pairs

$$
\left|\Psi_{n}\right\rangle \quad \text { and } \quad\left|\Psi_{n}^{T}\right\rangle=\hat{T}\left|\Psi_{n}\right\rangle .
$$

Therefore, if all we know about the state of the system is the energy $E_{n}$, Kramers degeneracy prevents the very existence of 
nonzero local spin polarization,

$$
\operatorname{Tr} \hat{\rho}_{n} \hat{\sigma}_{j}=0 \quad \text { for } \quad \hat{\rho}_{n} \sim\left|\Psi_{n}\right\rangle\left\langle\Psi_{n}|+| \Psi_{n}^{T}\right\rangle\left\langle\Psi_{n}^{T}\right| .
$$

To understand this we note that, if the state of the system is given by an incoherent (statistical) superposition corresponding to a microcanonical preparation at energy $E_{n}$, any observable $\hat{O}$ odd under time reversal

$$
\hat{T}^{-1} \hat{O} \hat{T}=-\hat{O}
$$

automatically satisfies

$$
\operatorname{Tr} \hat{\rho}_{n} \hat{O}=0 .
$$

Such an ensemble of random mixed states (as the one in Ref. [8]) gives nontrivial results only for correlations of the local charge, as this is the only density with nonzero expectation value with respect to $\hat{\rho}_{n}$.

However, if besides the energy $E_{n}$ we also know for certain that the system is in a pure (albeit unknown) state, any specific (but random) superposition

$$
\left|\psi_{n}^{a, b}\right\rangle=a\left|\Psi_{n}\right\rangle+b\left|\Psi_{n}^{T}\right\rangle, \quad|a|^{2}+|b|^{2}=1
$$

shows indeed a very rich spatial and spin structure, which depends on the particular choice of the energy index $n$ and the coefficients $(a, b)$. Once an ensemble of pure states is constructed, universal correlations should emerge after averaging over $n,(a, b)$ and this is the subject we are interested in here. The average over $a, b$ on top of the usual average over energy used in the case of scalar waves reflects our intrinsic ignorance about the pure state when all we specify is its energy. Other types of ensembles can be constructed where more information about the system is available, thus, fixing the ambiguity and selecting a particular combination of the Kramers pairs at each energy, but with the price of breaking TRI, and therefore we will not discuss such option here.

Physical realizations of an ensemble of coherent superpositions (as achieved by a preparation method that chooses randomly a particular linear combination of the Kramers pair) will be discussed in Sec. III. Assuming for the moment that such a preparation mechanism exists, in Fig. 1(a) we show the local expectation value of $\hat{\sigma}_{z}$ for a typical member of the ensemble, and our goal here is to present a theoretical
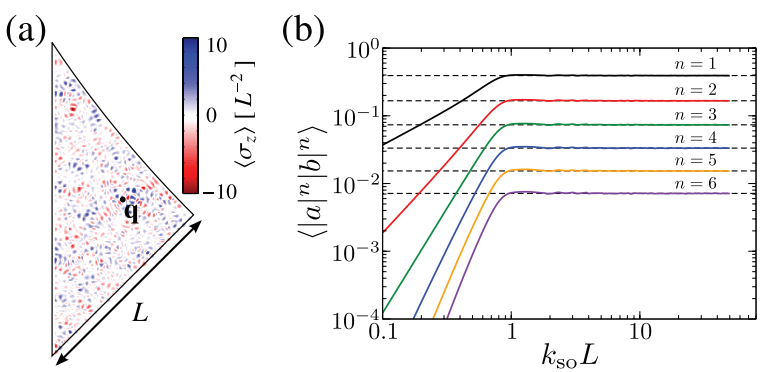

FIG. 1. (Color online) (a) Spin polarization in $z$ direction in units of $L^{-2}$ for the 400th eigenstate of the "star billiard" after a measurement of the local spin in $z$ direction at position $\mathbf{q}$, with $k_{\mathrm{so}} L=10$. (b) Spatial average of $\left\langle|a(\mathbf{q})|^{n}|b(\mathbf{q})|^{n}\right\rangle$ for the local coefficients $a(\mathbf{q}), b(\mathbf{q})$ in Eq. (11) as a function of $k_{\mathrm{so}} L$. Dashed lines: average of $|a|^{n}|b|^{n}$ as given by the uniform distribution on the unit sphere. approach to understand and predict the spatial statistics of such an imprinted pattern.

It is important to stress that in order to have an ensemble of pure random states that inherits the same symmetries of the Hamiltonian in Eq. (1) (as any sensible definition of the ensemble should do), an essential ingredient is that the average over the random complex variables $(a, b)$ must be performed with respect to the flat measure. The reason is that TRI of the ensemble as a whole requires that all pure states $\left|\psi_{n}^{a, b}\right\rangle$ for arbitrary $(a, b)$ satisfying $|a|^{2}+|b|^{2}=1$ must be equally likely.

\section{PHYSICAL AND NUMERICAL IMPLEMENTATION}

We discuss now a possible mechanism that generates the ensemble of pure spinors $\left|\psi_{n}^{a, b}\right\rangle$. For our construction we only need to apply an external field on the electron gas such that the superposition of the $n$th Kramers pair locally satisfies

$$
\psi_{n}(\mathbf{q}, s)=\mathcal{N} \delta_{s, \uparrow},
$$

at a given position $\mathbf{q}$ and for some constant $\mathcal{N}$ fixed by normalization. Such effects appear, for example, by a projective measurement of the local spin polarization in $z$ direction, by a contact with a spin-polarized STM, or by the presence of a single magnetic impurity, and in all these cases we need to assure that the physical interaction leading to the boundary condition Eq. (9) is strictly local; otherwise, it would lift the degeneracy of the Kramers doublet. Figure 1(a) corresponds to the choice of $\mathbf{q}$ indicated inside the cavity.

In order to fulfill the condition in Eq. (9), the coefficients $(a, b)$ must have a precise form $a(\mathbf{q}), b(\mathbf{q})$ depending on the location $\mathbf{q}$ and on the energy $E_{n}$ (not indicated). This form is found by demanding

$$
a(\mathbf{q}) \Psi_{n}(\mathbf{q}, s)+b(\mathbf{q}) \Psi_{n}^{T}(\mathbf{q}, s)=\mathcal{N} \delta_{s, \uparrow},
$$

which gives

$$
\begin{aligned}
& a(\mathbf{q})=\frac{\Psi_{n}(\mathbf{q}, \uparrow)^{*}}{\sqrt{\left|\Psi_{n}(\mathbf{q}, \uparrow)\right|^{2}+\left|\Psi_{n}(\mathbf{q}, \downarrow)\right|^{2}}}, \\
& b(\mathbf{q})=-\frac{\Psi_{n}(\mathbf{q}, \downarrow)}{\sqrt{\left|\Psi_{n}(\mathbf{q}, \uparrow)\right|^{2}+\left|\Psi_{n}(\mathbf{q}, \downarrow)\right|^{2}}} .
\end{aligned}
$$

From Eq. (11) we see that an ensemble of random coefficients (and correspondingly an ensemble of random spinors) can be constructed by variation of the index $n$, the position $\mathbf{q}$, or both as long as $\Psi_{n}(\mathbf{q}, \uparrow), \Psi_{n}(\mathbf{q}, \downarrow)$ behave as random variables when the energy $E_{n}$ and/or the position $\mathbf{q}$ are sampled.

The choice of keeping $\mathbf{q}$ fixed, and varying $E_{n}$ inside some energy window, will lead to an anisotropic ensemble that is physically realized by implementing Eq. (9) as an extra boundary condition for Eq. (1). We are not going to consider this situation here, as we want to study the effect of confinement by hard walls as the only mechanism breaking spatial isotropy and, therefore, in the following we will consider averages over both $\mathbf{q}$ and $E_{n}$. The first required to generate random coefficients $(a, b)$, the second needed to gain statistical significance.

What is left is to check whether, for fixed energy $E_{n}$, the ensemble coefficients $a(\mathbf{q}), b(\mathbf{q})$ indeed behave like random 
variables when the position $\mathbf{q}$ is varied, with the only correlation given by $|a|^{2}+|b|^{2}=1$. This we do by explicit numerical calculation of the statistical moments of the distribution $P(a, b)$.

For the numerical simulations we consider the desymmetrized "star billiard" [shown in Fig. 1(a)], which is characterized by only one parameter (the radius of the circular arc in units of the length $L$ of the short straight segment). For $k_{\text {so }}=0$, this billiard is known to display hard chaos in the classical limit and, therefore, it is expected to exhibit universality in the spatial correlations of its quantum eigenstates [10]. We explicitly diagonalize the Hamiltonian using an iterative technique [11] in shift-and-invert mode [12], which gives the corresponding Kramers pair for each eigenenergy $E_{n}$.

For fixed $E_{n}$, any local function $\mathcal{F}\left[\psi_{n}^{a, b}(\mathbf{r}, s), \psi_{n}^{a, b}\left(\mathbf{r}^{\prime}, s^{\prime}\right)\right]$ of the state will fluctuate when $\mathbf{q}$ is randomly chosen inside the billiard. Also, in chaotic systems it is expected that for strong SOI, spin randomization takes place and the spatial fluctuations of the state carry over to the local coefficients $a(\mathbf{q}), b(\mathbf{q})$, leading to a universal distribution. In Fig. 1(b) we study numerically how this universality is achieved with increasing $k_{\text {so }} L$. We find [13]

$$
\left\langle|a(\mathbf{q})|^{n}|b(\mathbf{q})|^{n}\right\rangle \sim\left(k_{\mathrm{so}} L\right)^{n} \quad \text { for } \quad n \leqslant 4 \text { and } k_{\mathrm{so}} L<1 .
$$

As is further shown in Fig. 1(b), for larger $k_{\text {so }} L$ we get

$$
\left\langle|a(\mathbf{q})|^{n}|b(\mathbf{q})|^{n}\right\rangle=\int_{|a|^{2}+|b|^{2}=1}|a|^{n}|b|^{n} \quad \text { for } \quad k_{\mathrm{so}} L>1 .
$$

Therefore, as long as $k_{\mathrm{so}} L>1$ we can replace the average over $(a, b)$ on the unit sphere by an average over $\mathbf{q}$,

$$
\begin{gathered}
\int_{|a|^{2}+|b|^{2}=1} \mathcal{F}\left[\psi_{n}^{a, b}(\mathbf{r}, s), \psi_{n}^{a, b}\left(\mathbf{r}^{\prime}, s^{\prime}\right)\right] d a d b \\
=\int_{\Omega} \mathcal{F}\left[\psi_{n}^{a(\mathbf{q}), b(\mathbf{q})}(\mathbf{r}, s), \psi_{n}^{a(\mathbf{q}), b(\mathbf{q})}\left(\mathbf{r}^{\prime}, s^{\prime}\right)\right] d \mathbf{q} .
\end{gathered}
$$

In this way, we realize for $k_{\mathrm{so}} L>1$ the formal ensemble of random spinors $\left|\psi_{n}^{a, b}\right\rangle$ as an ensemble $\left|\psi_{n}^{a(\mathbf{q}), b(\mathbf{q})}\right\rangle$ given by randomly changing the position where a physical interaction (projective measurement, STM tip or local magnetic impurity) enforces the condition Eq. (9).

For the numerical calculations presented from now on, we use the numerical Kramers pair $\left(\left|\Psi_{n}\right\rangle,\left|\Psi_{n}^{T}\right\rangle\right)$ to construct the expectation values of local observables $\mathcal{F}\left[\psi_{n}^{a, b}(\mathbf{r}, s), \psi_{n}^{a, b}\left(\mathbf{r}^{\prime}, s^{\prime}\right)\right]$ with respect to pure random states solving Eq. (1) at fixed energy and given $(a, b)$. In a second step, the average over $(a, b)$ on the unit sphere is performed exactly. Finally, we proceed in the usual way one studies spatial fluctuations of wavefunctions in classically chaotic systems, namely, we use the exact numerical results for local observables to perform an energy average where we expect universality to emerge.

\section{THE TWO-POINT CORRELATION FUNCTION: UNIVERSAL RESULTS AND BOUNDARY EFFECTS}

Any theory that attempts to describe the universality of spatial correlations must deal with two different aspects: first, the issue of the universal behavior of the amplitude correlator both in the bulk and near a boundary, and second, the appropriate description of correlators beyond the bilinear form in the amplitudes.

The original approaches to universal spatial fluctuations in chaotic systems considered these two phenomena to share the same origin (as both can be derived from Berry's ansatz stating that wavefunctions of classically chaotic systems behave as random superpositions of plane waves [10]). Later it was recognized [14] that for scalar waves $\chi_{n}(\mathbf{r})$ the two-point correlator, defined as the average of the product of amplitudes at different positions,

$$
R\left(\mathbf{r}, \mathbf{r}^{\prime} ; E \simeq E_{n}\right)=\left\langle\chi_{n}(\mathbf{r}) \chi_{n}(\mathbf{r})^{*}\right\rangle,
$$

is actually an intrinsically microscopic object that can be derived without any further assumption from the exact Green function,

$$
\hat{G}^{ \pm}(E)=\left(\hat{H}-E \pm i 0^{+}\right)^{-1},
$$

by means of the formula

$$
R\left(\mathbf{r}, \mathbf{r}^{\prime} ; E\right) \simeq \frac{\Delta(E)}{2 \pi i}\left\langle G^{-}\left(\mathbf{r}, \mathbf{r}^{\prime} ; E\right)-G^{+}\left(\mathbf{r}, \mathbf{r}^{\prime} ; E\right)\right\rangle .
$$

As mentioned before, here $\langle\ldots\rangle$ denotes ensemble and energy average and $\Delta(E)$ is the mean level spacing at energy $E$. For systems where the quantum state is represented by an object with several components $\Psi(\mathbf{r}, s)$ (as in our case where the extra index $s$ labels spin direction) this result is directly generalized by considering a matrix-valued correlation $C\left(\mathbf{r}, \mathbf{r}^{\prime} ; E\right)$ with entries given by

$\left[C\left(\mathbf{r}, \mathbf{r}^{\prime} ; E\right)\right]_{s, s^{\prime}} \simeq \frac{\Delta(E)}{2 \pi i}\left\langle\left[G^{-}\left(\mathbf{r}, \mathbf{r}^{\prime} ; E\right)\right]_{s, s^{\prime}}-\left[G^{+}\left(\mathbf{r}, \mathbf{r}^{\prime} ; E\right)\right]_{s, s^{\prime}}\right\rangle$,

in terms of the matrix-valued Green function. In the following, every mention to the two-point correlation function will refer to its matrix-valued representation in spin space.

For systems with SOI and away from the boundary, the exact Green function is approximated by its bulk value to get the universal prediction for the bulk,

$$
C_{0}\left(\mathbf{r}, \mathbf{r}^{\prime} ; E\right)=\frac{1}{4 k}\left[\begin{array}{cc}
C_{D}(d ; E) & -e^{-i \theta} C_{S}(d ; E) \\
e^{i \theta} C_{S}(d ; E) & C_{D}(d ; E)
\end{array}\right],
$$

where

$$
\begin{gathered}
C_{D}(d ; E)=k_{+} J_{0}\left(k_{+} d\right)+k_{-} J_{0}\left(k_{-} d\right), \\
C_{S}(d ; E)=k_{+} J_{1}\left(k_{+} d\right)-k_{-} J_{1}\left(k_{-} d\right),
\end{gathered}
$$

and $J_{n}(x)$ are Bessel functions. We further defined

$$
k_{ \pm}=\sqrt{k^{2}+k_{\mathrm{so}}^{2}} \pm k_{\mathrm{so}}, \quad \text { with } \quad k=\sqrt{2 m E / \hbar^{2}}
$$

and

$$
\mathbf{r}-\mathbf{r}^{\prime}=d(\cos \theta, \sin \theta) .
$$

The result, Eq. (17), can be also obtained using a modified Berry ansatz including SOI, as in Refs. [8,15].

To go beyond the results for the bulk, we use a multiple reflection expansion to construct the matrix-valued Green function near a wall, assumed to be an infinite straight line at $y=0$ [16]. Translational invariance in the $x$ direction suggests performing a Fourier transform (indicated by a tilde) from 
$x-x^{\prime}$ to $k_{x}$. As explained in more detail in the Appendix, the method provides a closed form for the boundary contribution (for notational convenience the dependence on $E$ is kept implicit)

$$
\tilde{G}_{1}\left(k_{x}, y, y^{\prime}\right)=2 \partial \tilde{G}_{0}\left(k_{x}, y\right)\left[1-2 \partial \tilde{G}_{0}\left(k_{x}, 0\right)\right]^{-1} \tilde{G}_{0}\left(k_{x}, y^{\prime}\right),
$$

in terms of the bulk Green function

$$
\tilde{G}_{0}\left(k_{x}, y-y^{\prime}\right)=\sum_{+,-}( \pm) \frac{e^{-a_{ \pm}\left|y-y^{\prime}\right|}}{2 a_{ \pm}}\left(f_{ \pm} \hat{I}-g \hat{\sigma}_{y}+h \hat{\sigma}_{x}\right)
$$

and its normal derivatives $\partial \tilde{G}_{0}$ at the boundary (see Appendix). Here we introduced

$$
f_{ \pm}=g\left(k^{2}-k_{ \pm}^{2}\right) / k_{\mathrm{so}} \quad \text { and } \quad h=i g a_{ \pm} \operatorname{sgn}\left(y-y^{\prime}\right)
$$

with

$$
g=k_{x} /\left(2 \sqrt{k^{2}+k_{\mathrm{so}}^{2}}\right) \text { and } a_{ \pm}=\sqrt{k_{x}^{2}-k_{ \pm}^{2}}
$$

where the branch of the square root is defined to be $\operatorname{Re} a_{ \pm}>0$.

In order to construct the correlation function in real space, the inverse Fourier transform of $\tilde{G}_{1}$ is calculated in stationary phase approximation, well justified in the regime $k L \gg 1$. As shown in the Appendix, inverse Fourier transform of $\tilde{G}_{0}\left(k_{x}, y-y^{\prime}\right)$ leads to a linear combination of expressions with phases of the form

$$
\Phi\left(k_{x}\right)=\sqrt{k_{ \pm}^{2}-k_{x}^{2}} y+\sqrt{k_{ \pm}^{2}-k_{x}^{2}} y^{\prime}+k_{x}\left(x-x^{\prime}\right) .
$$

Therefore, the geometry of the saddle points $\Phi^{\prime}\left(k_{x}\right)=0$ is a deformed version of the Snell law, with two (instead of just one) possibilities for the incoming and outgoing wavevectors corresponding to two $k_{ \pm}$.

In a first attempt, the limit $k_{\mathrm{so}} / k \rightarrow 0, k_{\mathrm{so}} L \rightarrow$ const. where the SOI is not included for the stationary phase condition can be considered, effectively recovering the usual Snell law and referred to in the following as the "one-beam method." Although the one-beam method allows for analytical treatment leading to compact expressions (explicitly shown in the Appendix), it provides insufficient results for the spatial correlations, and we use instead a "two-beams" approach [18] based on the consistent solution of the stationary phase conditions for each independent combination of beams up to second order in $k_{\text {so }} / k$, therefore differentiating between $k_{+}$ and $k_{-}$.

In Fig. 2 we illustrate how the choice of method can dramatically affect the results, in particular for correlations involving charge current and spin densities. The figure depicts universal spatial correlations found using results of Sec. V where the one-beam [Fig. 2(a)] and two-beam [Fig. 2(b)] methods are employed. Noticeable differences appear due to interferences between the two incoming and outgoing wavevectors $k_{ \pm}$, which are lost if the one-beam method is used. The comparison shown in Figs. 3, 4, and 5 between numerically exact correlators (calculated using the exact eigenstates of the system) and the universal predictions based on the two-point correlation function confirms the need to use the (considerably more involved) two-beam method.

After the two-beam method is implemented, the effect of the hard wall on the spatial correlations is then incorporated as a contribution $C_{1}$ on top of the bulk result $C_{0}$, Eq. (17).

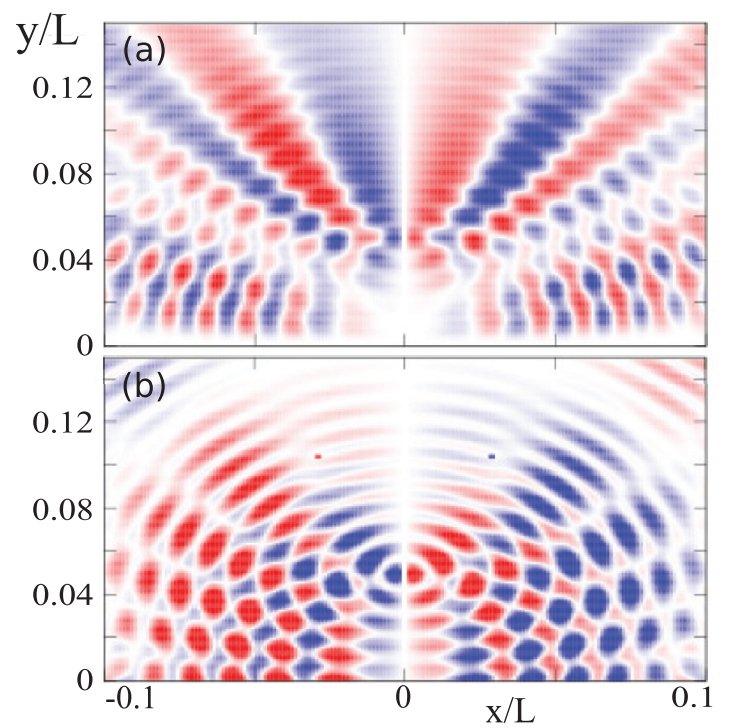

FIG. 2. (Color online) Comparison of the one-beam (a) and twobeam (b) methods for the calculation of correlation functions near a hard wall. Shown is the spatial correlator $C_{J_{x} \sigma_{z}}\left(\mathbf{r}, \mathbf{r}^{\prime} ; E\right)$ between the spin current in $y$ direction at point $\mathbf{r}$ and the spin density in $z$ direction at position $\mathbf{r}^{\prime}=(0,0.05 L)$ as a function of $\mathbf{r}=(x, y)$, near a wall at $y=0$. For comparison with numerical results see Fig. 5 .

The calculation of the boundary contribution $G_{1}$ to the total green function [used together with Eq. (15) to get $C_{1}$ ] is straightforward but tedious. Its main steps and final results

(a)
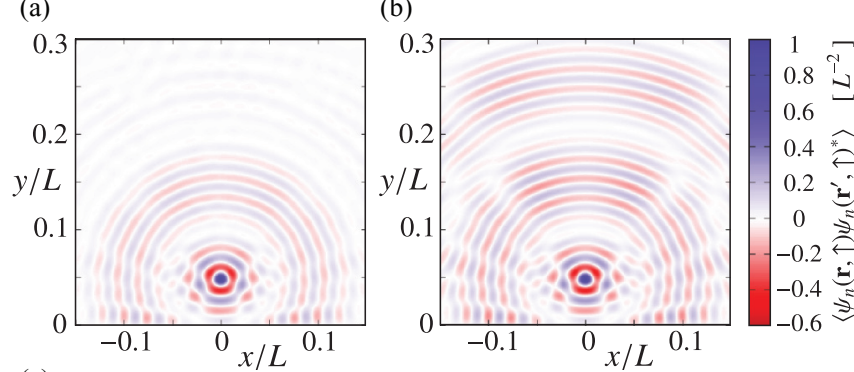

(c)

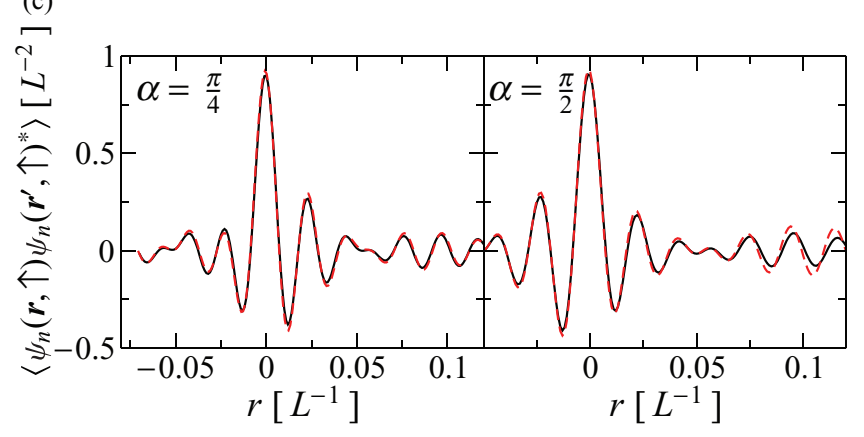

FIG. 3. (Color online) Comparison (in units of $L^{-2}$ ) between the numerically obtained (a) spatial two-point correlation function $\left\langle\psi_{n}(\mathbf{r}, \uparrow) \psi_{n}\left(\mathbf{r}^{\prime}, \uparrow\right)^{*}\right\rangle$ (defined by ensemble and energy average over 300 states near $n=3000$ ) as a function of $\mathbf{r}$, near a wall at $y=0$ [with $\left.\mathbf{r}^{\prime}=(0,0.05 L)\right]$ and the universal analytical results $C_{0}^{\uparrow, \uparrow}\left(\mathbf{r}, \mathbf{r}^{\prime} ; E\right)+$ $C_{1}^{\uparrow, \uparrow}\left(\mathbf{r}, \mathbf{r}^{\prime} ; E\right)(\mathrm{b})$; see text. The lower panel (c) shows radial cuts of the figure along the lines $\alpha=\pi / 4,2 \pi / 4$ with solid lines displaying the numerical calculations and dashed lines displaying the theoretical prediction. We use $k_{\mathrm{so}} / k=0.1$ and $k L=300$. 
(a)

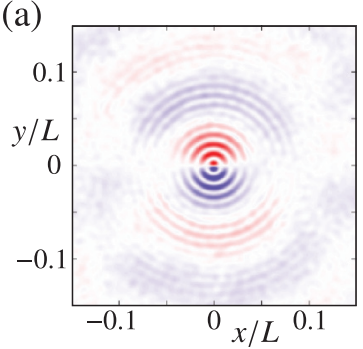

(c)

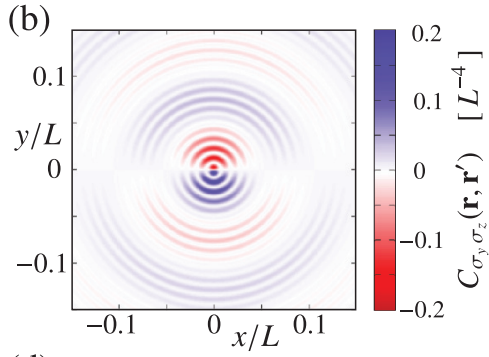

(d)

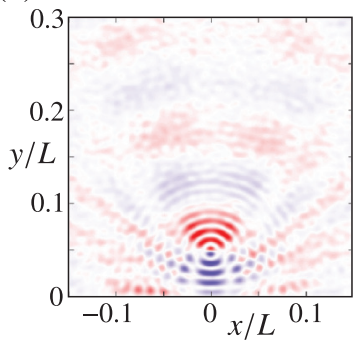

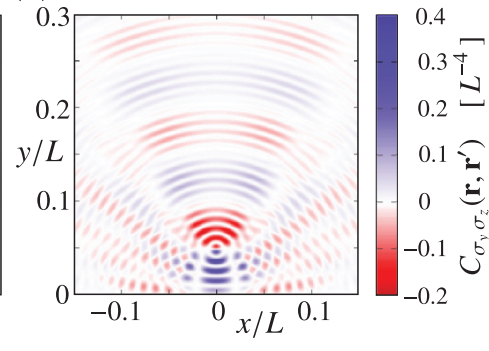

FIG. 4. (Color online) Spatial correlation $C_{\sigma_{y} \sigma_{z}}\left(\mathbf{r}, \mathbf{r}^{\prime} ; E\right.$ ) (in units of $L^{-4}$ ) of spin densities in the bulk (a,b) and near a wall at $y=0$ (c,d) as function of r. Panels (a) and (c): results of Eq. (22) based on numerically obtained eigenstates. Panels (b) and (d): universal prediction $C_{\sigma_{y} \sigma_{z}}^{\mathrm{SRWM}}\left(\mathbf{r}, \mathbf{r}^{\prime} ; E\right)$, Eq. (24). We use $\mathbf{r}^{\prime}=(0,0.05 L), k_{\mathrm{so}} / k=$ 0.1 , and $k L=300$. Averages are calculated using 300 Kramers pairs around $E_{3000}$.

for both the one-beam and two-beam methods are presented in the Appendix.

The effect of a nearby boundary on the two-point amplitude correlator is depicted in Fig. 2, showing excellent agreement

(a)

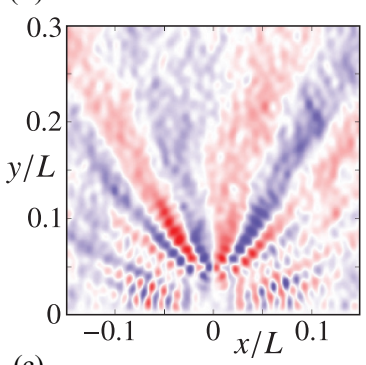

(b)

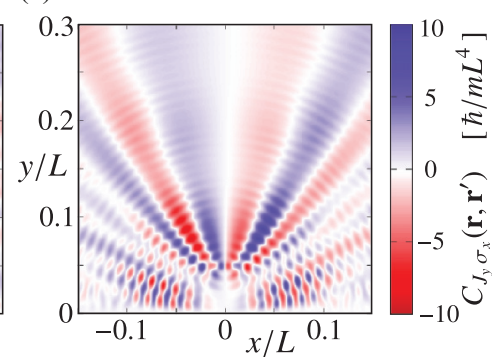

(c)

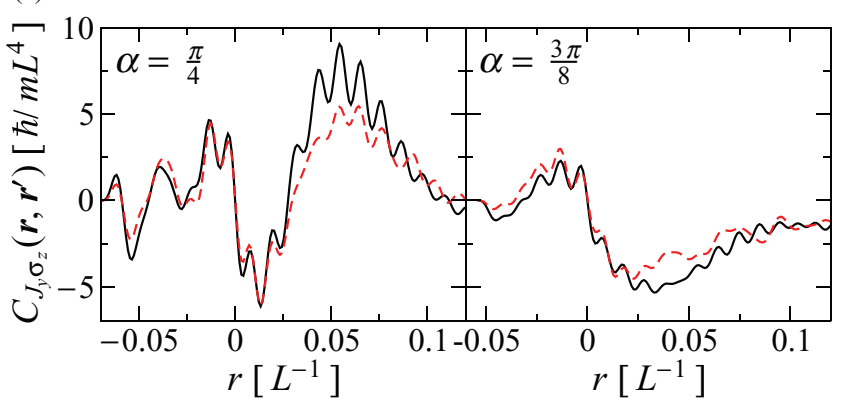

FIG. 5. (Color online) Spatial correlation $C_{J_{y} \sigma_{z}}\left(\mathbf{r}, \mathbf{r}^{\prime} ; E\right)$ (in units of $\hbar m^{-1} L^{-4}$ ) between spin current and spin density near a wall at $y=0$ produced, for instance, by applying a spin current with $y$ polarization at $\mathbf{r}^{\prime}=(0,05 \mathrm{~L})$ and measuring the dependence with $\mathbf{r}$ of the spin polarization in $z$ direction. Left side (a): numerical calculation from Eq. (22). Right side (b): universal prediction $C_{J_{y} \sigma_{z}}^{\mathrm{SRWM}}\left(\mathbf{r}, \mathbf{r}^{\prime} ; E\right)$ from Eq. (24). The conventions and parameters are the same as described in the legend of Fig. 3.

between numerical simulations and our analytical (but very lengthy) formula. This boundary effect is the spatial analog of the perimeter term in the Weyl formula [19] for the smooth part of the density of states of a system with SOI [18].

We have checked that changes on the size of the energy window, relative positions $\mathbf{r}, \mathbf{r}^{\prime}$, position of the wall and SOI strength do not affect the quality of our results for all the entries of the correlation matrix. Therefore we conclude that spatial correlations of spinor amplitudes are described by the formula (15), and universality emerges when the Green function can be approximated by its universal limit for the bulk or near a hard wall. That this is precisely the case for chaotic quantum systems was shown for the scalar case in [20], and the same argument (that after average paths with multiple reflections produce sub-dominant effects) holds here as well.

An important issue when one discusses universality of spatial correlations is the interplay between energy average, long range correlations and non-universal effects, and we address briefly this point here. Besides its linear size $(L)$ the system has two scales, namely the de Broglie wavelenght $\lambda=2 \pi / k$ and the spin precession length $\lambda_{\mathrm{so}}=2 \pi / k_{\mathrm{so}}$, and correspondingly the correlation function presents both short scale (de Broglie) and large scale (spin-orbit) oscillations. In order to make claims of universality both of then must be visible and we must study the spatial dependence of the correlation functions on scales larger than $\lambda_{\text {so }}$. However, the universality of the two-point correlation function is a consequence of the dominance of short paths in the Green function, as the direct path gives the bulk term and a path hitting the nearest boundary just once gives the boundary correction. For a given Kramers pair with fixed energy $E_{n}$, the effect of the short paths will be hidden among the contribution of the infinite number of paths joining $\mathbf{r}$ with $\mathbf{r}^{\prime}$, and the correlation function obtained by averaging over $(a, b)$ will be strongly dependent on the particular pair under consideration. It is a well-known fact that emergence of universality requires extra averaging to supress the incoherent oscillations coming from (essentially random) directions due to long trajectories in favor of the direct and short path contributions [10].

There are two extra average mechanisms at hand to diminish the effect of long paths to the correlation function, namely a spatial average around $\mathbf{r}$ or $\mathbf{r}^{\prime}$ (or both) or an energy average within the window $E_{n} \in[E-W / 2, E+W / 2]$. By a stationary phase argument, the spatial average is known to reduce the combined effect of nondirect trajectories to a factor $1 / k L$ [20] compared with the amplitude $1 / \sqrt{k\left|\mathbf{r}-\mathbf{r}^{\prime}\right|}>$ $1 / \sqrt{k L}$ of the direct path. Spatial averages over regions of order $\lambda$ are then very effective to check the direct path contribution to the correlation function. However, by their very definition, a spatial average destroy completely the information about the location of the boundaries, and therefore it is not suitable to study boundary effects. For this purpose, the energy average is more convenient, as all the eigenfunctions inside the energy window satisfy the same boundary conditions.

Semiclassically, the effect of the energy average is to damp contributions to the Green function coming from paths larger than the length scale $L_{W}=\lambda \sqrt{E / W}$, and in order to make negligible the effect of long paths hitting the boundary several times we need to choose our parameters such that $L_{W} \simeq L$. These considerations show that the energy average will also 
affect the universal correlation function for separations of the order of the system linear size. In particular, in order to observe enough spin oscillations we need separations of the order $\left|\mathbf{r}-\mathbf{r}^{\prime}\right| \simeq \lambda_{\text {so }}$, but our choice of parameters $\lambda_{\text {so }} \simeq 10 \lambda$ and $k L=300$ gives $L / \lambda_{\text {so }} \simeq 5$, resulting in a loss of accuracy for the long-range tail of the universal correlations when nonuniversal effects are eliminated by an energy average. Therefore, delicate long-range deviations of higher-order correlations characteristic of the crossover regimes (as shown in Ref. [21] for the orthogonal-to-unitary transition) cannot be excluded based on our numerical results.

\section{SPATIAL CORRELATIONS BETWEEN SPIN, CHARGE, AND CURRENT DENSITIES}

We now turn our attention to the experimentally more relevant case of spatial correlations for local densities. We consider observables of the form

$$
\hat{A}^{d}(\mathbf{r})=\delta(\hat{\mathbf{r}}-\mathbf{r}) \otimes \hat{A} .
$$

For given position $\mathbf{r}$, the choice $\hat{A}=\hat{I}$ describes the local charge density, while $\hat{A}=\hat{\sigma}_{i}$ gives the spin density in $i$ th direction. Introducing the spinor $\psi_{n}^{a, b}(\mathbf{r})=\left\langle\mathbf{r} \mid \psi_{n}^{a, b}\right\rangle$, the numerical spatial density correlations are then constructed from the numerical eigenstates by ensemble and energy average

$$
C_{A B}\left(\mathbf{r}, \mathbf{r}^{\prime} ; E\right)=\left\langle\left[\psi_{n}^{a, b}(\mathbf{r})^{\dagger} \hat{A} \psi_{n}^{a, b}(\mathbf{r})\right]\left[\psi_{n}^{a, b}\left(\mathbf{r}^{\prime}\right)^{\dagger} \hat{B} \psi_{n}^{a, b}\left(\mathbf{r}^{\prime}\right)\right]\right\rangle .
$$

Following a well-established procedure in systems without spin, we assume that the spinor amplitudes have Gaussian fluctuations [14], and we replace the energy and ensemble averages by a single average over a functional distribution of spinor fields,

$$
C_{A B}^{\mathrm{SRWM}}\left(\mathbf{r}, \mathbf{r}^{\prime} ; E\right)=\int P(\psi) \psi(\mathbf{r})^{\dagger} \hat{A} \psi(\mathbf{r}) \psi\left(\mathbf{r}^{\prime}\right)^{\dagger} \hat{B} \psi\left(\mathbf{r}^{\prime}\right) \mathcal{D}[\psi] .
$$

In this spin random wave model (SRWM), the probability distribution $P(\psi)$ is Gaussian and therefore uniquely given by its two-point matrix-valued correlation function

$$
\int P(\psi) \psi(\mathbf{r}) \psi\left(\mathbf{r}^{\prime}\right)^{\dagger} \mathcal{D}[\psi]=C\left(\mathbf{r}, \mathbf{r}^{\prime} ; E\right),
$$

which we replace by the universal amplitude correlator based on the microscopic Green function.

Having at hand a Gaussian theory with known two-point correlators, we can decouple averages over higher-order functionals of the state by straightforward use of Wick's theorem. For the particular case of local observables, this gives [18]

$$
\begin{aligned}
C_{A B}^{\mathrm{SRWM}}\left(\mathbf{r}, \mathbf{r}^{\prime} ; E\right)= & \operatorname{Tr}\left[\hat{A} C\left(\mathbf{r}, \mathbf{r}^{\prime} ; E\right) \hat{B} C\left(\mathbf{r}^{\prime}, \mathbf{r} ; E\right)\right] \\
& +\operatorname{Tr}[\hat{A} C(\mathbf{r}, \mathbf{r} ; E)] \operatorname{Tr}\left[\hat{B} C\left(\mathbf{r}^{\prime}, \mathbf{r}^{\prime} ; E\right)\right],
\end{aligned}
$$

where the trace is over spin variables only.

Equation (24) and its interpretation as describing an ensemble of pure random states solving Eq. (1) allows us to derive universal results for spatial correlations of local observables after inserting the expressions $C_{0}, C_{1}$ of the correlator $C\left(\mathbf{r}, \mathbf{r}^{\prime} ; E\right)$ for the bulk or its modification near the wall. In order to check the underlying Gaussian assumption, in
Figs. 3, 4, and 5 we compare the result, Eq. (24), with numerical results based on Eq. (22). We find considerable agreement even for the subtle patterns emerging from interference effects due to the boundary. In order to make it more quantitative, we present also a comparison of the numerical and analytical results along the radial direction of the $2 \mathrm{D}$ plots in Figs. 3, 4, and 5 for two different angles $\alpha=\pi / 4,3 \pi / 8$.

\section{CONNECTION WITH LINEAR RESPONSE THEORY}

The physical relevance of $C_{A B}$ can be extended beyond its statistical interpretation by noticing that the connected part of $C_{A B}^{\mathrm{SRWM}}$ at the Fermi energy $E=E_{F}$ can be rigorously related through

$$
\lim _{w \rightarrow 0} \int_{-\infty}^{\infty} \phi_{A B}(t) \frac{\mathrm{e}^{i w t}}{w} d t=i \operatorname{Tr}\left[\hat{A} C\left(\mathbf{r}, \mathbf{r}^{\prime} ; E_{F}\right) \hat{B} C\left(\mathbf{r}^{\prime}, \mathbf{r} ; E_{F}\right)\right]
$$

to the dc component of the dynamical response function $\phi_{A B}\left(t-t^{\prime}\right)$, defined by

$$
\phi_{A B}\left(t-t^{\prime}\right) \propto\left\langle\left[\hat{A}^{d}(\mathbf{r}, t), \hat{B}^{d}\left(\mathbf{r}^{\prime}, t^{\prime}\right)\right]\right\rangle,
$$

describing the change of the expectation value of $\hat{A}^{d}$ at time $t$ when an infinitesimal perturbation affects the system at time $t^{\prime}$ through a coupling with the observable $\hat{B}^{d}$ [22]. Equation (25) relates an experimentally accessible quantity, the response function, with the correlator quantifying the statistical fluctuations of the random spinor field.

\section{CONCLUSIONS}

To summarize, we have shown that pure states of electrons in confined chaotic geometries in the presence of strong spinorbit coupling exhibit spatial spin and spin current correlations given by the universal correlations of a Gaussian random spinor field [23]. We have carefully analyzed the effect of time reversal invariance leading to Kramers degeneracy and have shown that the correct interpretation of such a model demands as extra ingredient the condition of purity for each member of the ensemble. We have also shown that this condition is implemented by averaging over random linear combinations of the Kramers pair for each energy and presented physical mechanisms performing such random preparation for realistic situations.

Our results can be applied to a large class of correlators both in the bulk and near a boundary. They hold not only for the correlations between amplitudes but also for spatial correlations of spin densities and spin currents, more generally for any pair of local observables, and display very good agreement when compared with numerically exact simulations.

Finally, the physical meaning of the results obtained within the statistical approach is further elucidated by a rigorous identity relating linear response coefficients to the universal correlators, opening a straightforward possibility to measure such correlations.

\section{ACKNOWLEDGMENTS}

We acknowledge several helpful questions of an anonymous referee leading, e.g, to the statistical analysis in Fig. 1(b). This 
work was supported by the Deutsche Forschungsgemeinschaft within SFB 689 (J.D.U., K.R.) and by the funds of the Erdal İnönü chair and TUBA GEBIP (I.A.). I.A. thanks the University of Regensburg for their hospitality.

\section{APPENDIX : GREEN FUNCTION FOR THE RASHBA 2DEG NEAR A HARD WALL}

Here we present the main steps in the derivation of the Green function for a $2 \mathrm{D}$ electron gas in the presence of spin-orbit interaction of Rashba type near a flat, hard wall modeled as a Dirichlet boundary condition along $y=0$. We follow Ref. [6] where a multiple reflection expansion in coordinate space for systems with spin-orbit interaction was constructed, and we now explicitly evaluate it in real space for the particular case of the infinite straight wall. A mixed representation for the later case was also considered in Ref. [24].

From now on we will refer always to the retarded Green's function if not declared otherwise. Usually the exact Green's function satisfying a certain boundary condition is not known. But one can always write the exact Green's function as a sum of the free-space Green's function $G_{0}\left(\mathbf{r}, \mathbf{r}^{\prime} ; E\right)$ and a correction term $G_{1}\left(\mathbf{r}, \mathbf{r}^{\prime} ; E\right)$ with which the exact Green's function satisfies the boundary condition:

$$
G\left(\mathbf{r}, \mathbf{r}^{\prime} ; E\right)=G_{0}\left(\mathbf{r}, \mathbf{r}^{\prime} ; E\right)+G_{1}\left(\mathbf{r}, \mathbf{r}^{\prime} ; E\right) .
$$

In the following derivation we will use units such that $\hbar^{2} / 2 m=1$. For a Dirichlet boundary condition on an arbitrary surface $S$ of a volume $V$ the correction $G_{1}\left(\mathbf{r}, \mathbf{r}^{\prime} ; E\right)$ must satisfy the following differential equation and boundary condition:

$$
\begin{aligned}
(\hat{H}-E) G_{1} & =0, \text { for } \mathbf{r} \text { in } V, \\
G_{1} & =-G_{0}, \text { for } \mathbf{r} \text { on } S .
\end{aligned}
$$

For the case without spin-orbit coupling we can apply the method of images and obtain

$$
G_{1}\left(\mathbf{r}, \mathbf{r}^{\prime} ; E\right)=G_{1}\left(\mathbf{r}, \mathbf{r}_{i} ; E\right),
$$

where $\mathbf{r}_{i}$ is the image of $\mathbf{r}^{\prime}$ on the boundary outside the billiard. But this is not applicable for the case of spin-orbit coupling. The reason is that the Green's function for the path from the image point $\mathbf{r}_{i}$ does not coincide with the Green's function for the path that is reflected at the surface. Therefore, we will use a different approach to obtain the correction term $G_{1}$. We will use the so-called multiple reflection method. Originally derived by Balian and Bloch [19] for a scalar Greens function, it was later extended to particle and hole degrees of freedom in Ref. [25]. Here we use its extension to spin degrees of freedom.

The main idea of the multiple reflection expansion consists of replacing the differential equation and boundary condition, Eq. (A2), by a two-dimensional integral equation on the boundary. For a Dirichlet boundary condition this is achieved by representing $G_{1}$ as a double-layer potential in terms of an unknown density $\mu$. The density $\mu$ can be obtained by solving the following integral equation [6]:

$$
\begin{aligned}
\mu\left(\beta, \mathbf{r}^{\prime} ; E\right)= & 2 G_{0}\left(\beta, \mathbf{r}^{\prime} ; E\right) \\
& +2 \int_{S} \partial_{\alpha} G_{0}(\beta, \alpha ; E) \mu\left(\alpha, \mathbf{r}^{\prime} ; E\right) d \sigma_{\alpha}
\end{aligned}
$$

and has a well-defined unique solution. In the following, greek indexes $\alpha, \beta, \ldots$ label points $\mathbf{r}(\alpha), \mathbf{r}(\beta), \ldots$ along the boundary $S$ with $d \sigma_{\alpha}, d \sigma_{\beta}, \ldots$ the associated differential surface element.

Equation (A4) is an integral equation of the first kind that can be solved by iteration and leads to a series that is convergent for any finite imaginary part of the energy argument. This procedure yields the following expression for the correction term $G_{1}$ :

$$
\begin{aligned}
G_{1}\left(\mathbf{r}, \mathbf{r}^{\prime} ; E\right)= & 2 \int_{S} \partial_{\alpha} G_{0}(\mathbf{r}, \alpha ; E) G_{0}\left(\alpha, \mathbf{r}^{\prime} ; E\right) d \sigma_{\alpha} \\
& +2^{2} \int_{S} \int_{S} \partial_{\alpha} G_{0}(\mathbf{r}, \alpha ; E) \partial_{\beta} G_{0}(\alpha, \beta ; E) \\
& \times G_{0}\left(\beta, \mathbf{r}^{\prime} ; E\right) d \sigma_{\alpha} d \sigma_{\beta}+\ldots,
\end{aligned}
$$

where

$$
\partial_{\alpha} G_{0}(\mathbf{r}, \alpha ; E)=\left.\hat{\mathbf{n}}_{\alpha} \cdot \nabla_{r^{\prime}} G_{0}\left(\mathbf{r}, \mathbf{r}^{\prime} ; E\right)\right|_{\mathbf{r}^{\prime}=\mathbf{r}(\alpha)},
$$

with $\hat{\mathbf{n}}_{\alpha}$ the (inner) unit vector at the boundary point parametrized by $\alpha$, is the normal derivative of $G_{0}\left(\mathbf{r}, \mathbf{r}^{\prime} ; E\right)$ at the point on the boundary $\mathbf{r}^{\prime}=\mathbf{r}(\alpha)$. Equation (A5) may be interpreted as a multiple reflection expansion for a wave starting from $\mathbf{r}^{\prime}$, which is reflected on $S$ at the points $\mathbf{r}(\alpha), \mathbf{r}(\beta), \mathbf{r}(\gamma), \ldots$ and finally goes to $\mathbf{r}$.

For two-dimensional systems and points close to the surface, we can replace the boundary by a straight wall. We then perform a one-dimensional Fourier transform along the boundary and obtain the complete Green's function $G$ in a mixed representation of momentum and spatial coordinates. The Green's function in mixed representation reads:

$$
\begin{aligned}
\tilde{G}\left(k_{x}, y, y^{\prime} ; E\right)= & \tilde{G}_{0}\left(k_{x}, y, y^{\prime} ; E\right)+2 \partial \tilde{G}_{0}\left(k_{x}, y ; E\right) \\
& \times\left[1-2 \partial \tilde{G}_{0}\left(k_{x} ; E\right)\right]^{-1} \tilde{G}_{0}\left(k_{x}, y^{\prime} ; E\right),
\end{aligned}
$$

where

$$
\begin{aligned}
\tilde{G}_{0}\left(k_{x}, y^{\prime} ; E\right) & =\int G_{0}\left(x=0, y=0, x^{\prime}, y^{\prime} ; E\right) e^{-i k_{x} x^{\prime}} d x^{\prime}, \\
\partial \tilde{G}_{0}\left(k_{x} ; E\right) & =\left.\int \frac{\partial G_{0}\left(x=0, y, x^{\prime}, y^{\prime} ; E\right)}{\partial y^{\prime}}\right|_{y=y^{\prime}=0} e^{-i k_{x} x^{\prime}} d x^{\prime} .
\end{aligned}
$$

Note that $\tilde{G}$ only depends on the free-space Green's function $G_{0}$ in mixed representation. However, to apply the random wave model, we need the two-point correlation function and, thus, the full Green's function in coordinate space. To obtain $G\left(\mathbf{r}, \mathbf{r}^{\prime} ; E\right)$, we first have to calculate the free-space Green's function in mixed representation, in order to solve Eq. (A7). We then transform $\tilde{G}\left(k_{x}, y, y^{\prime} ; E\right)$ back to coordinate space. For the following derivation of the free Rashba Green's function in mixed representation, we write the Rashba Hamiltonian Eq. (1) in a slightly different form:

$$
\hat{H}=\frac{\hat{p}^{2}}{2 m} \hat{I}+\alpha \hat{\mathbf{p}} \cdot \hat{\eta},
$$

where $\hat{\eta}$ is an operator in spin space, defined as

$$
\hat{\eta}=\mathbf{e}_{z} \times \hat{\sigma}=\left(-\hat{\sigma}_{y}, \hat{\sigma}_{x}, 0\right) .
$$

Here, $\hat{\sigma}_{x}$ and $\hat{\sigma}_{y}$ are Pauli matrices and $\mathbf{e}_{z}=(0,0,1)$, the unit vector in $z$ direction. It turns out to be easier to start with the Rashba Green's function in momentum space and perform 
the Fourier transform in one component of the momentum to obtain the Green's function in mixed representation. Defining

$$
G_{0}\left(\mathbf{r}, \mathbf{r}^{\prime} ; E\right)=\frac{1}{(2 \pi)^{2}} \int \tilde{G}_{0}(\mathbf{p} ; E) e^{\frac{i}{\hbar} \mathbf{p}\left(\mathbf{r}-\mathbf{r}^{\prime}\right)} d \mathbf{p},
$$

and using the translational invariance, we obtain for the freespace Green's function in momentum space:

$$
\begin{aligned}
\tilde{G}_{0}(\mathbf{p} ; E+i \epsilon)= & \frac{E+i \epsilon-\frac{p^{2}}{2 m}}{\left(E+i \epsilon-\frac{p^{2}}{2 m}\right)^{2}-\alpha^{2} p^{2}} I \\
& +\frac{\alpha}{\left(E+i \epsilon-\frac{p^{2}}{2 m}\right)^{2}-\alpha^{2} p^{2}} \mathbf{p} \cdot \eta,
\end{aligned}
$$

where we have used that $(\mathbf{p} \cdot \eta)^{2}=p^{2}$. It is convenient to introduce the wave number $\hbar \mathbf{k}=\mathbf{p}$ and to use again units such that $\hbar^{2} / 2 m=1$. Then we obtain

$$
\begin{aligned}
\tilde{G}_{0}\left(\mathbf{k}, k_{E}\right)= & \frac{k_{E}^{2}-k^{2}}{\left(k_{E}^{2}-k^{2}\right)^{2}-4 k_{\mathrm{so}}^{2} k^{2}} I \\
& +\frac{2 k_{\mathrm{so}}}{\left(k_{E}^{2}-k^{2}\right)^{2}-4 k_{\mathrm{so}}^{2} k^{2}} \mathbf{k} \cdot \eta,
\end{aligned}
$$

where we have denoted by $k_{E}$ the square root of $E+i \epsilon$, which has a positive imaginary part:

$$
k_{E}=\sqrt{E+i \epsilon}, \quad \operatorname{Im}\left(k_{E}\right)>0 .
$$

For the further calculations we define:

$$
\begin{aligned}
& a_{ \pm}\left(k_{x}\right)=\sqrt{k_{x}^{2}-k_{ \pm}^{2}(E)} \text { for } k_{x} \geqslant k_{ \pm}, \\
& a_{ \pm}\left(k_{x}\right)=-i \sqrt{k_{ \pm}^{2}(E)-k_{x}^{2}} \text { for } k_{x}<k_{ \pm} .
\end{aligned}
$$

Since $k_{E}^{2}$ has a positive imaginary part, the function $a_{ \pm}\left(k_{x}\right)$ is a continuous univalued function for real $k_{x}$. The determination chosen in Eq. (A15) goes from $-i k_{ \pm}$to $+\infty$ following the contour indicated in Fig. 3 of Ref. [19], as $k_{x}$ varies from 0 to $+\infty$. The values of $a_{ \pm}\left(k_{x}\right)$ for $k_{x}$ varying from 0 to $+\infty$ have a positive real part and a negative imaginary part.

After some algebra, we obtain:

$$
\begin{aligned}
\tilde{G}_{0}\left(k_{x}, k_{y}, k_{E}\right)= & \left(\frac{1}{k_{y}^{2}+a_{+}^{2}}-\frac{1}{k_{y}^{2}+a_{-}^{2}}\right) \\
& \times\left[\frac{k_{E}^{2}-k_{x}^{2}-k_{y}^{2}}{4 k_{\mathrm{so}} \sqrt{k_{E}^{2}+k_{\mathrm{so}}^{2}}} I+\frac{\mathbf{k} \cdot \eta}{2 \sqrt{k_{E}^{2}+k_{\mathrm{so}}^{2}}}\right] .
\end{aligned}
$$

This is now a suitable expression to perform the partial Fourier transform from $k_{y}$ to $y-y^{\prime}$ :

$$
\tilde{G}_{0}\left(k_{x}, y-y^{\prime} ; E\right)=\frac{1}{2 \pi} \int \tilde{G}_{0}\left(k_{x}, k_{y} ; E\right) e^{i k_{y}\left(y-y^{\prime}\right)} d k_{y} .
$$

This integral can be solved by integrating in complex space. The residual of the integrand is given by

$$
\operatorname{Res}\left[\frac{f\left(k_{y}\right) e^{i k_{y}\left(y-y^{\prime}\right)}}{k_{y}^{2}+a^{2}}\right]_{ \pm i a}=\frac{f( \pm i a) e^{\mp a\left(y-y^{\prime}\right)}}{ \pm 2 i a},
$$

for a well-behaving function $f\left(k_{y}\right)$. Since $\operatorname{Re}\left(a_{ \pm}\right)>0$ the pole $+i a_{ \pm}$lies in the upper complex half plane and the pole $-i a_{ \pm}$ in the lower one. To make the integral convergent we have to integrate in the upper half plane for $\left(y-y^{\prime}\right)>0$ and in the lower half plane for $\left(y-y^{\prime}\right)<0$. Taking into account the direction of integration for these two cases, we finally get

$$
\frac{1}{2 \pi} \int \frac{f\left(k_{y}\right) e^{i k_{y}\left(y-y^{\prime}\right)}}{k_{y}^{2}+a^{2}} d k_{y}=\frac{f\left[i a \cdot \operatorname{sgn}\left(y-y^{\prime}\right)\right] e^{-a\left|y-y^{\prime}\right|}}{2 a} .
$$

After some organizing, we obtain

$$
\begin{aligned}
\tilde{G}_{0}\left(k_{x}, y-y^{\prime} ; E\right)= & \frac{k_{E}^{2}-k_{+}^{2}}{4 k_{\mathrm{so}} \sqrt{k_{E}^{2}+k_{\mathrm{so}}^{2}}} \cdot \frac{e^{-a_{+}\left|y-y^{\prime}\right|}}{2 a_{+}} I+\frac{k_{E}^{2}-k_{-}^{2}}{4 k_{\mathrm{so}} \sqrt{k_{E}^{2}+k_{\mathrm{so}}^{2}}} \cdot \frac{e^{a_{-}\left|y-y^{\prime}\right|}}{2 a_{-}} I+\frac{k_{x}}{2 \sqrt{k_{E}^{2}+k_{\mathrm{so}}^{2}}}\left(\frac{e^{-a_{+}\left|y-y^{\prime}\right|}}{2 a_{+}}-\frac{e^{-a_{-}\left|y-y^{\prime}\right|}}{2 a_{-}}\right) \eta_{x} \\
& +\frac{i \cdot \operatorname{sgn}\left(y-y^{\prime}\right)}{2 \sqrt{k_{E}^{2}+k_{\mathrm{so}}^{2}}}\left(\frac{e^{-a_{+}\left|y-y^{\prime}\right|}}{2}-\frac{e^{-a_{-}\left|y-y^{\prime}\right|}}{2}\right) \eta_{y}
\end{aligned}
$$

We therefore have everything we need to calculate the correction term $G_{1}$ in mixed representation:

$$
\tilde{G}_{1}\left(k_{x}, y, y^{\prime} ; E\right)=2 \partial \tilde{G}_{0}\left(k_{x}, y ; E\right)\left[1-2 \partial \tilde{G}_{0}\left(k_{x} ; E\right)\right]^{-1} \tilde{G}_{0}\left(k_{x}, y^{\prime} ; E\right)
$$

where

$$
\begin{aligned}
\tilde{G}_{0}\left(k_{x}, y^{\prime} ; E\right)= & -\frac{1}{2}\left(\frac{e^{-a_{+} y^{\prime}}}{2 a_{+}}+\frac{e^{-a_{-} y^{\prime}}}{2 a_{-}}\right) I-\frac{k_{\mathrm{so}}}{2 \sqrt{k_{E}^{2}+k_{\mathrm{so}}^{2}}}\left(\frac{e^{-a_{+} y^{\prime}}}{2 a_{+}}-\frac{e^{-a_{-} y^{\prime}}}{2 a_{-}}\right) I+\frac{k_{x}}{2 \sqrt{k_{E}^{2}+k_{\mathrm{so}}^{2}}}\left(\frac{e^{-a_{+} y^{\prime}}}{2 a_{+}}-\frac{e^{-a_{-} y^{\prime}}}{2 a_{-}}\right) \eta_{x} \\
& -\frac{i}{2 \sqrt{k_{E}^{2}+k_{\mathrm{so}}^{2}}}\left(\frac{e^{-a_{+} y^{\prime}}}{2}-\frac{e^{-a_{-} y^{\prime}}}{2}\right) \eta_{y},
\end{aligned}
$$


and

$$
\begin{aligned}
\partial \tilde{G}_{0}\left(k_{x}, y ; E\right)= & \left.\frac{\partial G_{0}\left(k_{x}, y, y^{\prime} ; E\right)}{\partial y^{\prime}}\right|_{y^{\prime}=0}=-\frac{1}{2}\left(\frac{e^{-a_{+} y}}{2}+\frac{e^{-a_{-} y}}{2}\right) I-\frac{k_{\mathrm{so}}}{2 \sqrt{k_{E}^{2}+k_{\mathrm{so}}^{2}}}\left(\frac{e^{-a_{+} y}}{2}-\frac{e^{-a_{-} y}}{2}\right) I \\
& +\frac{k_{x}}{2 \sqrt{k_{E}^{2}+k_{\mathrm{so}}^{2}}}\left(\frac{e^{-a_{+} y}}{2}-\frac{e^{-a_{-} y}}{2}\right) \eta_{x}+\frac{i}{2 \sqrt{k_{E}^{2}+k_{\mathrm{so}}^{2}}}\left(\frac{a_{+} e^{-a_{+} y}}{2}-\frac{a_{-} e^{-a_{-} y}}{2}\right) \eta_{y} .
\end{aligned}
$$

Furthermore, we have

$$
\partial \tilde{G}_{0}\left(k_{x} ; E\right)=\left.\frac{\partial G_{0}\left(k_{x}, y, y^{\prime} ; E\right)}{\partial y^{\prime}}\right|_{y=y^{\prime}=0}=\frac{\left(a_{+}-a_{-}\right) i \eta_{y}}{4 \sqrt{k_{E}^{2}+k_{\mathrm{so}}^{2}}},
$$

so that the kernel of the correction term is given by

$$
1+2 \partial \tilde{G}_{0}\left(k_{x} ; E\right)=I-\frac{\left(a_{+}-a_{-}\right) i \eta_{y}}{2 \sqrt{k_{E}^{2}+k_{\mathrm{so}}^{2}}},
$$

of which the inverse is easily found.

Since we are interested in the spatial autocorrelation function, the goal is now to Fourier transform the mixed Green's function $\tilde{G}_{1}\left(k_{x}, y, y^{\prime} ; E\right)$ back to coordinate space to obtain $G_{1}\left(x, x^{\prime}, y, y^{\prime} ; E\right)$ :

$$
G_{1}\left(x, x^{\prime}, y, y^{\prime} ; E\right)=\frac{1}{2 \pi} \int_{-\infty}^{\infty} \tilde{G}_{1}\left(k_{x}, y, y^{\prime} ; E\right) e^{i k_{x} x} d k_{x} .
$$

For the general case $k_{\text {so }} \neq 0$, the integration gets cumbersome. The integrals we have to solve to perform the Fourier transform back to coordinate space are of the kind

$$
\int_{-\infty}^{+\infty} f\left(k_{x}\right) e^{i \Phi\left(k_{x}\right)} d k_{x}
$$

where

$$
\Phi\left(k_{x}\right)=\sqrt{k_{ \pm}^{2}-k_{x}^{2}} y+\sqrt{k_{ \pm}^{2}-k_{x}^{2}} y^{\prime}+k_{x} x .
$$

In the following, we will focus on systems with strong spinorbit coupling, i.e., $k_{\mathrm{so}} L \gg 1$, where $L$ is the characteristic length of the billiard. This holds for materials like InAs and $\mathrm{InSb}$, and quantum dots with linear sizes of $\sim 200 \mathrm{~nm}$. Thus, we can assume the integrand to be highly oscillating and solve the Fourier integrals in the semiclassical limit with the stationary phase approximation. To find the stationary points $k_{x}^{*}$ we have to solve

$$
\left.\frac{\partial}{\partial k_{x}}\left(\sqrt{k_{ \pm}^{2}-k_{x}^{2}} y+\sqrt{k_{ \pm}^{2}-k_{x}^{2}} y^{\prime}+k_{x} x\right)\right|_{k_{x}=k_{x}^{*}}=0
$$

for the four different combinations of incoming and outgoing waves that are reflected at the boundary. The condition to find the stationary points reads

$$
\frac{x-x^{\prime}}{k_{x}^{*}}=\frac{y}{\sqrt{k_{ \pm}^{2}-\left(k_{x}^{*}\right)^{2}}}+\frac{y^{\prime}}{\sqrt{k_{ \pm}^{2}-\left(k_{x}^{*}\right)^{2}}} .
$$

This is nothing but Snell's law for a specific pair of incoming and outgoing waves to describe the relationship between the angles of incidence and refraction of a wave passing through a boundary between two different isotropic media. However, although this equation may look quite harmless, the square roots make its solution a rather complicated issue. Therefore, we will again consider the case of high energies, $\lambda=k_{\mathrm{so}} / k_{E} \ll 1$. Then we can expand Eq. (A26) for the stationary phase point in terms of $\lambda$ around 0 up to 2 nd order. We write the stationary phase point as

$$
k_{x}=k_{x}^{(0)}+\lambda k_{x}^{(1)}+\lambda^{2} k_{x}^{(2)}+\ldots,
$$

and also expand the prefactors and phases:

$$
\begin{aligned}
& \Phi\left(k_{x}\right)=\Phi\left(k_{x}^{(0)}\right)+\lambda \Phi^{\prime}\left(k_{x}^{(0)}, k_{x}^{(1)}\right)+\lambda^{2} \Phi^{\prime \prime}\left(k_{x}^{(0)}, k_{x}^{(1)}, k_{x}^{(2)}\right)+\ldots, \\
& f\left(k_{x}\right)=f\left(k_{x}^{(0)}\right)+\lambda f^{\prime}\left(k_{x}^{(0)}, k_{x}^{(1)}\right)+\lambda^{2} f^{\prime \prime}\left(k_{x}^{(0)}, k_{x}^{(1)}, k_{x}^{(2)}\right)+\ldots
\end{aligned}
$$

The individual terms for $k_{x}$ are then obtained by solving the expanded Eq. (A26) for each order of $\lambda$. Performing the stationary phase method with this approximation, we obtain the full Green's function $G\left(\mathbf{r}, \mathbf{r}^{\prime} ; E\right)$, which is used in Secs. IV and $\mathrm{V}$.

Explicitly, for the two-beam method, the boundary correction to the diagonal entries of the Green function reads

$$
\begin{aligned}
& G_{1, \uparrow \uparrow / \downarrow \downarrow} \\
& =\frac{(-1)^{3 / 4}}{32 \sqrt{k_{E}} \sqrt{2 \pi}\left(y+y^{\prime}\right)^{5}\left[\left(y+y^{\prime}\right)^{2}+\left(x-x^{\prime}\right)^{2}\right]^{5 / 4}} \\
& \times\left(\mp \frac{e^{\frac{1}{2} i[(\lambda-2) \lambda+2] k_{E}} \sqrt{\left(y+y^{\prime}\right)^{2}+\left(x-x^{\prime}\right)^{2}}\left(x-x^{\prime}\right)\left[\left(y+y^{\prime}\right)^{2}+\left(x-x^{\prime}\right)^{2}\right]\left\{[3 \lambda(3 \lambda-4)+8]\left(y+y^{\prime}\right)^{2}+8 \lambda^{2}\left(x-x^{\prime}\right)^{2}\right\}\left(y+y^{\prime}\right)^{3}}{y+y^{\prime} \mp i\left(x-x^{\prime}\right)}\right. \\
& \mp \frac{e^{\frac{1}{2} i[\lambda(\lambda+2)+2] k_{E} \sqrt{\left(y+y^{\prime}\right)^{2}+\left(x-x^{\prime}\right)^{2}}}\left(x-x^{\prime}\right)\left[\left(y+y^{\prime}\right)^{2}+\left(x-x^{\prime}\right)^{2}\right]\left\{[3 \lambda(3 \lambda+4)+8]\left(y+y^{\prime}\right)^{2}+8 \lambda^{2}\left(x+x^{\prime}\right)^{2}\right\}\left(y+y^{\prime}\right)^{3}}{y+y^{\prime} \mp i\left(x-x^{\prime}\right)} \\
& -e^{\frac{\left.i k_{E} \sqrt{\left(y+y^{\prime}\right)^{2}+\left(x-x^{\prime}\right)^{\prime}\left(\left(y+y^{\prime}\right)^{\prime}\right.}\left[([\lambda-2) \lambda+2] y+[\lambda(\lambda+2)+2] y^{\prime}\right)-4 \lambda^{2} y y^{\prime}\left(x-x^{\prime}\right)^{\prime}\right)}{2\left(y+y^{\prime}\right)^{4}}}\left\{8 i\left[y+y^{\prime} \pm i\left(x-x^{\prime}\right)\right]\left(y+y^{\prime}\right)^{6}\right. \\
& -4 i \lambda\left(y-y^{\prime}\right)\left[y+y^{\prime} \pm i\left(x-x^{\prime}\right)\right]\left[y+y^{\prime} \pm 2 i\left(x-x^{\prime}\right)\right]\left(y+y^{\prime}\right)^{4}
\end{aligned}
$$




$$
\begin{aligned}
& +\frac{\lambda^{2}\left[\left(y+y^{\prime}\right)^{2}+\left(x-x^{\prime}\right)^{2}\right]}{y+y^{\prime} \mp i\left(x-x^{\prime}\right)}\left[-3 i\left(y^{2}+6 y^{\prime} y+y^{\prime 2}\right)\left(y+y^{\prime}\right)^{4} \mp 12\left(y-y^{\prime}\right)^{2}\left(x-x^{\prime}\right)\left(y+y^{\prime}\right)^{3}\right. \\
& \left.\left.-56 i y y^{\prime}\left(x-x^{\prime}\right)^{2}\left(y+y^{\prime}\right)^{2} \mp 8\left(y^{2}-6 y^{\prime} y+y^{\prime 2}\right)\left(x-x^{\prime}\right)^{3}\left(y+y^{\prime}\right)-48 i y y^{\prime}\left(x-x^{\prime}\right)^{4}\right]\right\} \\
& -e^{\frac{i k_{E} \sqrt{\left(y+y^{\prime}\right)^{2}+\left(x-x^{\prime}\right)^{2}\left(\left(y+y^{\prime}\right)^{3}\left[\{\lambda(\lambda(2)+2)+2] y+[(\lambda-2) \lambda+2] y^{\prime} \mid-4 \lambda^{2} y y^{\prime}\left(x-x^{\prime}\right)^{2}\right)\right.}}{2\left(y+y^{\prime}\right)^{4}}} \\
& \times\left\{8 i\left[y+y^{\prime} \pm i\left(x-x^{\prime}\right)\right]\left(y+y^{\prime}\right)^{6}+4 i \lambda\left(y-y^{\prime}\right)\left[y+y^{\prime} \pm i\left(x-x^{\prime}\right)\right]\left[y+y^{\prime} \pm 2 i\left(x-x^{\prime}\right)\right]\left(y+y^{\prime}\right)^{4}\right. \\
& +\frac{\lambda^{2}\left[\left(y+y^{\prime}\right)^{2}+\left(x-x^{\prime}\right)^{2}\right]}{y+y^{\prime} \mp i\left(x-x^{\prime}\right)}\left[-3 i\left(y^{2}+6 y^{\prime} y+y^{\prime 2}\right)\left(y+y^{\prime}\right)^{4} \mp 12\left(y-y^{\prime}\right)^{2}\left(x-x^{\prime}\right)\left(y+y^{\prime}\right)^{3}\right. \\
& \left.\left.\left.-56 i y y^{\prime}\left(x-x^{\prime}\right)^{2}\left(y+y^{\prime}\right)^{2} \mp 8\left(y^{2}-6 y^{\prime} y+y^{\prime 2}\right)\left(x-x^{\prime}\right)^{3}\left(y+y^{\prime}\right)-48 i y y^{\prime}\left(x-x^{\prime}\right)^{4}\right]\right\}\right),
\end{aligned}
$$

where the upper sign corresponds to $G_{\uparrow \uparrow}$, and the lower sign corresponds to $G_{\downarrow \downarrow}$. For the off-diagonal elements we get

$$
\begin{aligned}
& G_{1, \uparrow \downarrow / \uparrow}=\frac{\left[-\frac{1}{\left(y+y^{\prime}\right)^{2}+\left(x-x^{\prime}\right)^{2}}\right]^{3 / 4}}{32 \sqrt{k_{E}} \sqrt{2 \pi}}\left(\mp \frac{e^{\left.\frac{1}{2} i[\lambda-2) \lambda+2\right] k_{E} \sqrt{\left(y+y^{\prime}\right)^{2}+\left(x-x^{\prime}\right)^{2}}}\left(x+x^{\prime}\right)\left\{[3 \lambda(3 \lambda-4)+8]\left(y+y^{\prime}\right)^{2}+8 \lambda^{2}\left(x+x^{\prime}\right)^{2}\right\}}{\left(y+y^{\prime}\right)^{2}}\right. \\
& \pm \frac{e^{\frac{1}{2} i[\lambda(\lambda+2)+2] k_{E} \sqrt{\left(y+y^{\prime}\right)^{2}+\left(x-x^{\prime}\right)^{2}}}\left(x-x^{\prime}\right)\left\{[3 \lambda(3 \lambda+4)+8]\left(y+y^{\prime}\right)^{2}+8 \lambda^{2}\left(x-x^{\prime}\right)^{2}\right\}}{\left(y+y^{\prime}\right)^{2}} \\
& -e^{\frac{i k_{E} \sqrt{\left(y+y^{\prime}\right)^{2}+\left(x-x^{\prime}\right)^{2}\left(\left(y+y^{\prime}\right)^{3}\right.}\left[([x-2) x+2] y+[\lambda(\lambda+2)+2] y^{\prime}-4 \lambda^{2} y y^{\prime}\left(x-x^{\prime}\right)^{2}\right)}{2\left(y+y^{\prime}\right)^{4}}} \\
& \times\left\{\frac{\left[-3 i\left(y^{2}+6 y^{\prime} y+y^{\prime 2}\right)\left(y+y^{\prime}\right)^{4}+12\left(y-y^{\prime}\right)\left(x-x^{\prime}\right)\left(y+y^{\prime}\right)^{4}+8\left(y-y^{\prime}\right)\left(x-x^{\prime}\right)^{3}\left(y+y^{\prime}\right)^{2}\right] \lambda^{2}}{\left(y+y^{\prime}\right)^{5}}\right. \\
& \left.-\frac{\left[72 i y y^{\prime}\left(x-x^{\prime}\right)^{2}\left(y+y^{\prime}\right)^{2}-48 i y y^{\prime}\left(x-x^{\prime}\right)^{4}\right] \lambda^{2}}{\left(y+y^{\prime}\right)^{5}}-4 i\left[y-y^{\prime}-2 i\left(x-x^{\prime}\right)\right] \lambda+8 i\left(y+y^{\prime}\right)\right\} \\
& +e^{\frac{i k_{E} \sqrt{\left(y+y^{\prime}\right)^{2}+\left(x-x^{\prime}\right)^{2}}\left(\left(y+y^{\prime}\right)^{3}\left[(\lambda(\lambda+2)+2] y+[\lambda-2) \lambda+2 y^{\prime} y^{\prime}-4 \lambda^{2} y y^{\prime}\left(x-x^{\prime}\right)^{2}\right)\right.}{2\left(y+y^{\prime}\right)^{4}}} \\
& \times\left\{-\frac{\left[3 i\left(y^{2}+6 y^{\prime} y+y^{\prime 2}\right)\left(y+y^{\prime}\right)^{4} \pm 12\left(y-y^{\prime}\right)\left(x-x^{\prime}\right)\left(y+y^{\prime}\right)^{4} \pm 8\left(y-y^{\prime}\right)\left(x-x^{\prime}\right)^{3}\left(y+y^{\prime}\right)^{2}\right] \lambda^{2}}{\left(y+y^{\prime}\right)^{5}}\right. \\
& \left.\left.+\frac{\left[72 i y y^{\prime}\left(x-x^{\prime}\right)^{2}\left(y+y^{\prime}\right)^{2}+48 i y y^{\prime}\left(x-x^{\prime}\right)^{4}\right] \lambda^{2}}{\left(y+y^{\prime}\right)^{5}}+4 i\left(y-y^{\prime}\right) \lambda \mp 8\left(x-x^{\prime}\right) \lambda \mp 8 i\left(y+y^{\prime}\right)\right\}\right),
\end{aligned}
$$

where the upper sign corresponds to $G_{\uparrow \downarrow}$, and the lower sign corresponds to $G_{\downarrow \uparrow}$.

For the sake of completeness we quote also the results for the boundary correction to the Green function when the one beam method is used. In this case we get

$$
G_{1, \uparrow \uparrow / \downarrow \downarrow}=\frac{e^{i k_{E} \sqrt{\left(x-x^{\prime}\right)^{2}+\left(y+y^{\prime}\right)^{2}}} \sqrt{4}-\frac{1}{\left(x-x^{\prime}\right)^{2}+\left(y+y^{\prime}\right)^{2}}}{4 \sqrt{k_{E}} \sqrt{2 \pi}\left(y+y^{\prime}\right)^{4}}\left[3 \lambda^{2}\left(x-x^{\prime}\right)^{4}+2 \lambda^{2}\left(y+y^{\prime}\right)^{2}\left(x-x^{\prime}\right)^{2}-\left(\lambda^{2}-2\right)\left(y+y^{\prime}\right)^{4}\right],
$$

and

$$
G_{1, \uparrow \downarrow / \downarrow \uparrow}=\mp \frac{\left(\frac{1}{4}-\frac{i}{4}\right) e^{i k_{E} \sqrt{\left(x-x^{\prime}\right)^{2}+\left(y+y^{\prime}\right)^{2}}} \lambda\left(x-x^{\prime}\right) \sqrt{4}\left(x-x^{\prime}\right)^{2}+\left(y+y^{\prime}\right)^{2}}{\sqrt{k_{E}} \sqrt{\pi}\left(y+y^{\prime}\right)^{2}},
$$

where the upper sign corresponds to $G_{\uparrow \downarrow}$, and the lower sign corresponds to $G_{\downarrow \uparrow}$.

[1] I. Zutic, J. Fabian, and S. D. Sarma, Rev. Mod. Phys. 76, 323 (2004)

[2] Y. K. Kato, R. C. Myers, A. C. Gossard, and D. D. Awschalom, Science 306, 1910 (2004); J. Wunderlich, B. Kaestner, J. Sinova, and T. Jungwirth, Phys. Rev. Lett. 94, 047204 (2005).

[3] J. Nitta, T. Akazaki, H. Takayanagi, and T. Enoki, Phys. Rev. Lett. 78, 1335 (1997); T. Schäpers, G. Engels, J. Lange, Th. Klocke, M. Hollfelder, and H. Lüth, J. Appl. Phys. 83, 4324 (1998).
[4] See for example, J. I. Inoue, G. E. W. Bauer, and L. W. Molenkamp, Phys. Rev. B 70, 041303 (2004).

[5] O. Zaitsev, D. Frustaglia, and K. Richter, Phys. Rev. Lett. 94, 026809 (2005); Ph. Jacquod, Nanotechnology 21, 274006 (2010).

[6] I. Adagideli, Ph. Jacquod, M. Scheid, M. Duckheim, D. Loss, and K. Richter, Phys. Rev. Lett. 105, 246807 (2010).

[7] E. N. Bulgakov and A. F. Sadreev, Phys. Rev. E 70, 056211 (2004). 
[8] A. T. Ngo, E. H. Kim, and S. E. Ulloa, Phys. Rev. B 84, 155457 (2011).

[9] J. J. Sakurai, Modern Quantum Mechanics (Prentice Hall, Upper Saddle River, NJ, 1993).

[10] M. V. Berry, J. Phys. A: Math. Gen. 10, 2083 (1977).

[11] R. B. Lehoucq, D. C. Sorensen, and C. Yang, ARPACK Users Guide: Solution of Large-Scale Eigenvalue Problems with Implicitly Restarted Arnoldi Methods (Society for Industrial and Applied Mathematics, Philadelphia, PA, 1998).

[12] For the solution of the sparse linear system arising in the shift-and-invert problem, we apply the MUMPS package: P. R. Amestoy, I. S. Duff, J. Koster, and J.-Y. LExcellent, SIAM J. Matrix Anal. Appl. 23, 15 (2001).

[13] In this regime the system effectively does not belong to the symplectic ensemble. See I. L. Aleiner and V. I. Falko, Phys. Rev. Lett. 87, 256801 (2001).

[14] S. Hortikar and M. Srednicki, Phys. Rev. Lett. 80, 1646 (1998); J. D. Urbina and K. Richter, J. Phys. A: Math. Gen. 36, L495 (2003),

[15] C. Lehner, project report, University of Regensburg (2004).

[16] If the confinement is realistically described by an array of atoms (a so called "quantum corral") instead of a straight wall, a multiple scattering approach similar to the one used here has been proposed in Ref. [17].

[17] J. D. Walls and E. J. Heller, Nano Lett. 7, 3377 (2007).

[18] D. Bauernfeind, diploma thesis, University of Regensburg (2008).

[19] R. Balian and C. Bloch, Ann. Phys. 60, 401 (1970).

[20] J. D. Urbina and K. Richter, Phys. Rev. E 70, 015201 (2004).

[21] H. J. Sommers and S. Iida, Phys. Rev. E 49, 2513 (1994); Y. H. Kim, U. Kuhl, H. J. Stöckmann, and P. W. Brouwer, Phys. Rev. Lett. 94, 036804 (2005).

[22] See, for example, K. Huang, Introduction to Statistical Physics (Taylor \& Francis, London, 2001).

[23] For geometries such that the classical motion is integrable (and therefore nonchaotic), the two-point correlator for both the bulk and near a boundary still takes the same universal form when the energy average does not discriminate among states with different symmetry. However, in the most relevant case, where the average is defined over subspaces with fixed good quantum numbers besides the energy, the correlator is strongly system-dependent.

[24] V. A. Zyuzin, P. G. Silvestrov, and E. G. Mishchenko, Phys. Rev. Lett. 99, 106601 (2007)

[25] I. Adagideli, and P. M. Goldbart, Int. J. Mod. Phys. B 16, 1381 (2002). 